\title{
Low-Cost Method for the Estimation of the Shape of Quadric Mirrors and Calibration of Catadioptric Cameras
}

\author{
Nuno Gonçalves and Helder Araújo \\ Institute of Systems and Robotics \\ Dept. of Electrical and Computers Engineering \\ University of Coimbra \\ Pinhal de Marrocos - POLO II - 3030 Coimbra \\ PORTUGAL \\ \{nunogon,helder\}@isr.uc.pt
}

Corresponding author: Nuno Gonçalves

Fax number: +351239406672

email: nunogon@isr.uc.pt

Keywords: mirror shape recovery, catadioptric imaging, camera pose estimation, mirror pose estimation 


\title{
Low-Cost Method for the Estimation of the Shape of Quadric Mirrors and Calibration of Catadioptric Cameras
}

\author{
Nuno Gonçalves and Helder Araújo \\ Institute of Systems and Robotics \\ Dept. of Electrical and Computers Engineering \\ University of Coimbra \\ Pinhal de Marrocos - POLO II - 3030 Coimbra \\ PORTUGAL \\ \{nunogon,helder\}@isr.uc.pt
}

\begin{abstract}
This paper tackles the problem of the quadric mirror estimation of omnidirectional imaging systems, as well as the poses of the camera relative to the mirror and to the world reference system. These estimates are obtained for quadric-shaped mirrors (including elliptic, parabolic, hyperbolic and spherical mirrors) where the position of the camera and mirror is unconstrained (fully non central configurations). The apparent contour of the mirror can be used to reduce the uncertainty in the estimation. Although it can enhance both the accuracy of the estimation (allowing the method to converge from farther initial configurations) and also its performance, its use is not strictly required. The intrinsic parameters of the camera model (pinhole or orthographic) are assumed to be known as well as the structure of a calibration object in local coordinates. The method is nonlinear and we use a genetic algorithm in conjunction with the Nelder-Mead simplex algorithm to minimize the objective function. Experimental results using real data are presented demonstrating the accuracy of this calibration method, comparing accuracy with and without the apparent contour and computing the reprojection error. As the mirror quadric is estimated, this method can also be used to identify and classify the mirror.
\end{abstract}

Keywords: mirror shape recovery, catadioptric imaging, camera pose estimation, mirror pose estimation 


\section{INTRODUCTION}

Changing the direction of light rays has been used for several applications and particularly it has been widely used for imaging purposes, usually with the help of mirrors or lenses. Panoramic imaging systems are very popular for applications requiring wide field of views. The systems that use mirrors and cameras are called catadioptric and recently they have been studied and used in several applications. Amongst all catadioptric systems, the most useful are those made up of rotationally symmetric mirrors. From these the most used are those whose mirrors are quadrics.

Catadioptric cameras can be divided into two types depending on whether the projection is central or not. The projection is central if all light rays intersect at a unique point. This point is called the effective viewpoint. In the general case the optical center of the camera is the effective viewpoint to guarantee central projection. Particularly, it has been shown by Nayar and Baker $[1,2]$ that for quadric mirror catadioptric systems, the central projection can be obtained only for a particular position of the camera optical center, usually the focus of the quadric. However, for the general case and when this constraint is relaxed, the projection is non central which implies that the light rays do not intersect each other at a single viewpoint. In that case, they are all tangent to a surface called caustic. The caustic of an imaging system can then be defined as the envelope of all reflected light rays that are imaged by a camera [3-5] and so it uniquely determines the imaging system. For central cameras, the caustic degenerates in a point.

This issue can be relevant since the geometry of central projection systems is much simpler. The projection model can be obtained in a closed form and generalized to all quadric mirror based systems. Geyer et al. [6, 7] and Barreto et al. [8] derived closed form expressions for central catadioptric systems. In addition, several algorithms for perspective cameras can be easily applied to central catadioptric systems [9-12]. Another classification of cameras was proposed recently in [13]. These General Linear Cameras include also catadioptric cameras for which all incident rays intersect the optical axis (all central catadioptric cameras and some non-central cameras, those where the mirror is aligned with the camera optical axis).

Since for many of new vision sensors their projection models are unknown, Grossberg and Nayar [14] introduced the black box camera model. For this model the correspondence between each image pixel and a 3D direction in space is estimated. The calibration of a vision system is then regarded as a list of correspondences between pixels and 3D rays in space. In [15] this calibration is assumed to be available to solve the problem of motion estimation and $3 \mathrm{D}$ reconstruction on a 
structure from motion basis. Ramalingam and Sturm [16] presented a generic method to calibrate any vision system based on the black box model. This method uses three images of a calibration pattern to recover the motion between the coordinate frames of the camera and of the objects. Its application to omnidirectional cameras is presented in [17] and subsequent work in this field includes $[18,19]$. Another approach to generalized cameras is proposed by Chen and Chang in [20] where they use an iterative model solve a classical NPnP problem to estimate the pose of the camera in relation to the world reference frame.

The problem of recognition, identification, classification and reconstruction of reflecting surfaces has been studied for many years and the approaches used are diverse. In the field of optics (considering reflectance models, polarization, color, photometric characteristics and structured light) there are some reference works like [21-29]. Savarese and Perona [30] have also formulated this problem by using fully calibrated cameras and studying the properties of a line projected on the mirror. They recover locally the shape of the mirror surface. Other approaches to the problem include stereo or multiple views [24, 31] and a moving observer or moving surfaces [32, 33].

Closer approaches to the problem include all works in catadioptric cameras, central or not, that use only geometric relations to the calibration, irrespective of the lighting and reflectance model, color, photometric or polarization issues. Calibration algorithms to central catadioptric cameras includes [34] where some geometric invariants are formulated and used to an accurate calibration of the intrinsic parameters. Projection of lines, spheres or a combination of both are used. A similar approach [35] uses the principal point to calibrate a central catadioptric camera using also geometric invariants. Other important work in central catadioptric cameras include [36, 37].

For non-central catadioptric cameras there are also calibration methods. Micusik and Pajdla $[38,39]$ have formulated a calibration method for near-central catadioptric cameras using two images in two steps. The first step assumed central projection and find the best suited parameters for the catadioptric system and in the second step the parameters are tuned to fit the non central model. Jeng and Tsai [40, 41] have used the particular case of hyperbolic-shaped mirror and have calibrated them to produce perspective images. Strelow et al. [42] have applied shape from motion to non central cameras. Gonçalves and Araújo have also presented calibration techniques to calibrate non central quadric mirror catadioptric cameras using correspondence between image points and incident light rays in space [43] using an adapted bundle adjustment technique and in [44] they have formulated a calibration technique based on two images and on collinearity of three points in the incident light rays. 
We are interested in catadioptric systems with quadric mirrors in which the camera is positioned in an unconstrained position relative to the mirror, thus performing a non central configuration. Our aim is to estimate the quadric mirror, and the poses of the camera relative to the mirror and to the world reference frame. The approach we present in this paper may or may not use the information from the apparent contour of the quadric (a conic) that may be visible or not. This information improves the method by both allowing for more accurate results (since the algorithm converges more easily to the solution) and also by improving its performance (allowing for a faster convergence). Notice that the apparent contour can be partially visible and one is still allowed to estimate its conic parameters. In the experiments we introduce a study on the influence of the apparent contour in the estimation.

In the next section the problem is stated and formalized, and the notation conventions are defined. The following section III discusses the use of the quadric apparent contour in the image plane in the calibration method and in section IV the nonlinear method is formalized by defining the objective/error function to be minimized. In section $\mathrm{V}$ the quadric mirror in its canonical form and the pose of the camera relative to the mirror are estimated from the result of the minimization algorithm (the quadric in camera coordinates). This also provides a means to classify the shape of the mirror. In the following section VI, the experimental tests using both synthetic data and real images are presented and, finally, in section VII a summary and the main conclusions of the work are presented.

\section{PROBLEM STATEMENT}

In this paper we consider a catadioptric camera made up by a camera (perspective or orthographic) and a quadric surface mirror. The camera is represented by its intrinsic parameters matrix $\mathbf{K}(3 \times 3)$ and the mirror surface by its quadric matrix $\mathbf{Q}(4 \times 4)$. The system is fully non-central so the location and pose of the camera relative to the mirror are unconstrained and represented by the screw rigid transformation $\mathbf{T}$ (a $4 \times 4$ matrix).

$\mathrm{Q}$ is expressed in the quadric coordinate system and in the camera coordinate system it is expressed by $\mathrm{Q}_{\text {cam }}=\mathrm{T}^{\mathrm{T}} \mathrm{QT}$. We also consider the quadric matrix given in its block form:

$$
\mathbf{Q}_{\text {cam }}=\left[\begin{array}{ll}
\mathbf{Q}_{\mathbf{3}_{\text {cam }}} & \mathbf{q}_{\mathrm{cam}} \\
\mathbf{q}_{\mathrm{cam}}{ }^{T} & q_{44_{\text {cam }}}
\end{array}\right]
$$


Consider now an object in the scene. Consider a set of 3D points $\mathbf{P}_{\mathbf{i}}$ with known coordinates in the object local reference frame (or in the world reference frame). This reference frame is related to the camera coordinate system by the screw rigid transformation $\mathbf{H}$ in such a way that $\mathbf{P}_{\text {icam }}=\mathbf{H P}_{\mathbf{i}}$.

The location and pose of the object relative to the camera (represented by the $4 \times 4$ matrix $\mathbf{H}$ ), is described by three rotations about the coordinate axes $\left(\theta_{X}, \theta_{Y}\right.$ and $\left.\theta_{Z}\right)$ and three translations along the same coordinate axes $\left(t_{X}, t_{Y}\right.$ and $\left.t_{Z}\right)$. See figure 1.

The goal of this paper is to describe a method for the estimation of the quadric surface $Q$, the pose of the camera relative to the mirror ( $\mathbf{T}$ ) and the pose of the set of points in the scene relative to the camera $(\mathbf{H})$.

\section{APPARENT CONTOUR}

The apparent contour of the quadric mirror contains useful information that can be used to estimate the quadric itself. It reduces the uncertainty in the estimation of the quadric elements.

As stated in [45], under the camera matrix $\mathbf{P}$ the conic $\mathbf{C}$ back-projects to the cone

$$
\mathrm{Q}_{\text {cone }}=\mathrm{P}^{\mathrm{T}} \mathrm{CP}
$$

where in the case of a perspective camera we have $\mathbf{P}=\mathbf{K}[\mathbf{I} \mid \mathbf{0}]$ and in this case it yields:

$$
\mathbf{Q}_{\text {cone }}=\left[\begin{array}{cc}
\mathbf{K}^{T} \mathbf{C K} & \mathbf{0} \\
\mathbf{0}^{T} & 0
\end{array}\right]
$$

On the other hand, [45] also states that the cone with vertex $\mathbf{V}$ and tangent to the quadric $\mathbf{Q}_{\text {cam }}$ is the degenerate quadric with the equation given by:

$$
\mathbf{Q}_{\text {cone }}=\left(\mathbf{V}^{T} \mathbf{Q}_{\text {cam }} \mathbf{V}\right) \mathbf{Q}_{\text {cam }}-\left(\mathbf{Q}_{\text {cam }} \mathbf{V}\right)\left(\mathbf{Q}_{\text {cam }} \mathbf{V}\right)^{T}
$$

and if the vertex is at the center of the coordinate system $\left(\mathbf{V}=\left[\begin{array}{llll}0 & 0 & 0 & 1\end{array}\right]\right)$ and the quadric matrix is expressed in block form, then the cone is given by:

$$
\mathbf{Q}_{\text {cone }}=\left[\begin{array}{cc}
q_{44_{\text {cam }}} \mathbf{Q}_{\mathbf{3}_{\text {cam }}}-\mathbf{q}_{\mathbf{c a m}} \mathbf{q}_{\mathbf{c a m}}{ }^{T} & \mathbf{0} \\
\mathbf{0} & 0
\end{array}\right]
$$


As a result it can be seen that the cones represented in equations 3 and 5 are projectively equivalent. That means that the following equations can be written:

$$
\left\{\begin{array}{l}
q_{44_{\mathrm{cam}}} q_{11_{\mathrm{cam}}}-q_{14_{\mathrm{cam}}} q_{14_{\mathrm{cam}}}=\Gamma_{11} \\
q_{44_{\mathrm{cam}}} q_{12_{\mathrm{cam}}}-q_{14_{\mathrm{cam}}} q_{24_{\mathrm{cam}}}=\Gamma_{12} \\
q_{44_{\mathrm{cam}}} q_{13_{\mathrm{cam}}}-q_{14_{\mathrm{cam}}} q_{34_{\mathrm{cam}}}=\Gamma_{13} \\
q_{44_{\mathrm{cam}}} q_{22_{\mathrm{cam}}}-q_{24_{\mathrm{cam}}} q_{24_{\mathrm{cam}}}=\Gamma_{22} \\
q_{44_{\mathrm{cam}}} q_{23_{\mathrm{cam}}}-q_{24_{\mathrm{cam}}} q_{34_{\mathrm{cam}}}=\Gamma_{23} \\
q_{44_{\mathrm{cam}}} q_{33_{\mathrm{cam}}}-q_{34_{\mathrm{cam}}} q_{34_{\mathrm{cam}}}=\Gamma_{33}
\end{array}\right.
$$

where $\Gamma_{i j}$ are the elements of the matrix $\Gamma=\mathbf{K}^{T} \mathbf{C K}$. These six equations correspond to five degrees of freedom since the matrices are symmetric and projectively equivalent. The five degrees of freedom can be represented independently by the five ratios. If the last equation is chosen as the reference, the following five equations are obtained:

$$
q_{i j_{c a m}}=\frac{\Gamma_{i j}\left(q_{44_{c a m}} q_{33_{c a m}}-q_{34_{c a m}} q_{34_{c a m}}\right)+\Gamma_{33} q_{i 4_{c a m}} q_{j 4_{c a m}}}{q_{44_{c a m}}}
$$

where $(i, j) \in\{(1,1),(1,2),(1,3),(2,2),(2,3)\}$. These equations define five of the quadric mirror parameters as function of the other five, as a function of the intrinsic parameters of the camera and also as a function of the conic that represents the quadric apparent contour.

\section{NONLINEAR CALIBRATION OF THE CATADIOPTRIC CAMERA}

The aim of this paper is to provide an algorithm to calibrate the mirror, its pose relative to the camera and the pose of the camera relative to 3D world coordinates. We assume that the intrinsic parameters of the perspective camera are known as well as the local structure of the 3D calibration scene points.

If the apparent contour (which is a conic in the image plane) of the quadric surface mirror is visible in the image it is possible to estimate its five independent parameters using at least five points (see for instance [45]). This conic is the $3 \times 3 \mathrm{C}$ matrix in equation 3 and since the intrinsic parameters $\mathbf{K}$ are known, the back-projected cone from the apparent contour of the mirror is known. On the other hand, this cone is projectively equivalent to the cone given by equation 5 , providing the five equations 7 that are used in our method. 
Additionally, as the quadric has nine degrees of freedom instead of the ten which is the number of parameters $q_{i j}$, the scale factor can be fixed if the value for one of parameters is arbitrarily chosen. We may choose for instance $q_{11}=1$ (however any other value or any other parameter can be chosen) which reduces the number of unknowns and also removes the scale factor ambiguity.

The algorithm we propose starts from an initial guess for all the ten unknown parameters (four for the quadric $\mathbf{Q}_{\mathbf{c a m}}-\left(q_{14}, q_{24}, q_{34}\right.$ and $\left.q_{44}\right)$ and six for the screw $\mathbf{H}$ - three translations and three rotation angles).

Consider the set of image points $\mathbf{p}_{\mathbf{i}}$ and their corresponding $3 \mathrm{D}$ coordinates $\mathbf{P}_{\mathbf{i}}$ in the local coordinate system of the calibration object. These 3D points have coordinates in the camera coordinate system given by $\mathbf{H P}_{\mathbf{i}}$. Back projecting image points to incident lines in space by using geometric optics is trivial (see for instance [38]) and gives a line $\mathbf{L}_{\mathbf{i}}$ for each image point of the set being considered.

The distance of a $3 \mathrm{D}$ point $\mathbf{P}$ to a line in space (defined by two points $\mathbf{A}$ and $\mathbf{B}$ ) is given by the following equation:

$$
d=\frac{\|B-A\| \times\|A-P\|}{\|B-A\|}
$$

where $\mathbf{A}, \mathbf{B}$ and $\mathbf{P}$ are expressed in inhomogeneous coordinates.

If the quadric $\mathrm{Q}_{\mathrm{cam}}$ and the transformation between the camera system and the local object system $\mathbf{H}$ are correct, points $\mathbf{H P}_{\mathbf{i}}$ belong to the lines $\mathbf{L}_{\mathbf{i}}$. However, in a wrong configuration, the lines will not pass through the $3 \mathrm{D}$ points $\mathbf{H P}_{\mathbf{i}}$. Thus, the distances between these points and the lines $\mathbf{L}_{\mathbf{i}}$ can be added to define the objective function to be minimized by any non-linear optimization method.

Once estimated, the three rotation angles and the three translations can be used to compute the transformation matrix $\mathbf{H}$ easily. This step is straightforward.

Although, uniqueness of the solution is not proved, in experiments it is in general achieved.

\section{QUADRIC POSE ESTIMATION}

In this section we describe how the quadric in its canonical form and its pose relative to the camera can be estimated starting from the quadric matrix computed in the camera coordinate frame. 
The general quadric can be described by a $4 \times 4$ symmetric matrix $Q_{\text {cam }}$ such that $\mathbf{X}^{\mathrm{T}} \mathrm{Q}_{\text {cam }} \mathrm{X}=\mathbf{0}$ holds for all points on the quadric surface (where $\mathbf{X}$ is the vector of the homogeneous coordinates of a surface point). Due to symmetry the quadric has ten coefficients and nine degrees of freedom.

If the quadric parameters $q_{11}$ to $q_{44}$ are computed using some known world structure (as stated by the previous sections) the corresponding quadric matrix can be diagonalized in such a way as to specify a change of coordinates transforming the quadric into its canonical form. That means that the coordinate transformation between the camera and the quadric coordinate frames can be estimated. As a result the misalignment between the camera and the mirror can be estimated. In $[46,47]$ an alternative form to convert the quadric into its canonical form is proposed, using block diagonalization $[48,49]$. However this method does not constrain the point transformation to be rigid as required in our case.

Consider a rigid transformation $\mathbf{T}$ made up by a rotation matrix $\mathbf{R}(3 \times 3)$ and a translation vector $\mathbf{t}$ and the quadric mirror in its reduced form given by equation 1 . These matrices can be written in the form:

$$
\mathbf{T}=\left[\begin{array}{ll}
\mathbf{R} & \mathbf{t} \\
\mathbf{0} & 1
\end{array}\right] \quad \mathbf{Q}=\left[\begin{array}{cc}
\mathbf{Q}_{3} & \mathbf{q} \\
\mathbf{q}^{\prime} & q_{44}
\end{array}\right]
$$

The generic quadric in the camera coordinate system $\left(Q_{\text {cam }}\right)$ is obtained through the application of the rigid transformation of the quadric $Q$ by $T$. The relationship is $Q_{\text {cam }}=T^{T} \mathbf{Q T}$. This equation can be expanded to obtain the following expression:

$$
\mathbf{Q}_{\text {cam }}=\left[\begin{array}{ll}
\mathbf{Q}_{\mathbf{3}} & \mathbf{q}_{\text {cam }} \\
\mathbf{q}_{\text {cam }}{ }^{T} & q_{44_{c a m}}
\end{array}\right]\left[\begin{array}{cc}
\mathbf{R}^{\mathbf{T}} \mathbf{Q}_{\mathbf{3}} \mathbf{R} & \mathbf{R}^{\mathbf{T}} \mathbf{Q}_{\mathbf{3}} \mathbf{t}+\mathbf{R}^{\mathbf{T}} \mathbf{q} \\
\mathbf{t}^{\mathbf{T}} \mathbf{Q}_{\mathbf{3}} \mathbf{R}+\mathbf{q}^{\mathbf{T}} \mathbf{R} & \mathbf{t}^{\mathbf{T}} \mathbf{Q}_{\mathbf{3}} \mathbf{t}+\mathbf{q}^{\mathbf{T}} \mathbf{t}+\mathbf{t}^{\mathbf{T}} \mathbf{q}+q_{44}
\end{array}\right]
$$

The goal is to estimate linearly the rigid transformation $\mathbf{T}$ and the quadric in its canonical form $\mathrm{Q}$, starting with the knowledge of the quadric $\mathbf{Q}_{\mathbf{c a m}}$. This estimation is impossible since there are more unknowns (twelve for the transformation and nine for the quadric) than equations. However, some constraints on the quadric $\mathrm{Q}$ allow the recovery of both $\mathrm{T}$ and $\mathrm{Q}$. The quadric mirror for the most general mirrors can also be expressed in the simpler form: 


$$
\mathbf{Q}=\left[\begin{array}{cccc}
1 & 0 & 0 & 0 \\
0 & 1 & 0 & 0 \\
0 & 0 & A & B / 2 \\
0 & 0 & B / 2 & -C
\end{array}\right]
$$

and so $\mathbf{Q}_{\mathbf{3}}$ is a diagonal matrix whose first two diagonal elements are unitary and $\mathbf{q}$ is a 3-vector whose first two components are zero. The mirrors that we wish to study are paraboloids, hyperboloids, ellipsoids and spheres. The constraints that the parameters must satisfy are: for paraboloids $(C=0 ; A=0)$, for ellipsoids $(B=0)$, for hyperboloids $(A<0 ; C<0)$ and for spheres $\left(A=1 ; C+B^{2} / 2>0\right)$. By changing the coordinates in the $Z$ axis for hyperboloids and spheres, the additional constraint $B=0$ can be applied. It can be seen that the parameter $B$ is non zero only for paraboloids and zero for all other mirror types and also that in the former case the parameter $A$ is zero. We thus have:

- paraboloids $-B \neq 0 ; A=0 ; C=0 \Longrightarrow \mathbf{Q}_{\mathbf{3}}=\left[\begin{array}{lll}1 & 0 & 0 \\ 0 & 1 & 0 \\ 0 & 0 & 0\end{array}\right], \mathbf{q}=\left[\begin{array}{lll}0 & 0 & B / 2\end{array}\right]^{T}$ and $q_{44}=0$

- hyperboloids, ellipsoids and spheres $-B=0 \Longrightarrow \mathbf{Q}_{\mathbf{3}}=\left[\begin{array}{lll}1 & 0 & 0 \\ 0 & 1 & 0 \\ 0 & 0 & A\end{array}\right]$ and $\mathbf{q}=\mathbf{0}^{T}$.

The estimation of the rotation matrix $\mathbf{R}$ and of the first block diagonal $\mathbf{Q}_{3}$ is performed using the diagonalization obtained by the eigenvalue decomposition of the matrix $Q_{3_{\text {cam }}}$. This step is simple and as a result we obtain two matrices such that $\mathbf{Q}_{\mathbf{3}_{\text {cam }}} \mathbf{V}_{\mathbf{3}}=\mathbf{V}_{\mathbf{3}} \mathbf{D}_{\mathbf{3}}$ where $\mathbf{D}_{\mathbf{3}}$ is a diagonal matrix with the eigenvalues in the principal diagonal and $V_{3}$ is a full matrix with their corresponding eigenvectors as columns. Due to the symmetric nature of the quadrics the eigenvectors matrices are naturally symmetric and therefore it can be written $\mathrm{Q}_{\mathbf{3}}$ cam $=\mathrm{V}_{\mathbf{3}} \mathrm{D}_{\mathbf{3}} \mathrm{V}_{\mathbf{3}}{ }^{T}$ since $\mathbf{V}_{\mathbf{3}}$ is orthogonal. Furthermore the diagonal matrix $\mathbf{D}_{\mathbf{3}}$ can be decomposed in such a way that the central matrix has the elements in the order required in the diagonal as stated in equation 11 . It thus yields: 


$$
\mathbf{D}_{\mathbf{3}}=\left[\begin{array}{ccc}
d_{1} & 0 & 0 \\
0 & d_{2} & 0 \\
0 & 0 & d_{3}
\end{array}\right]=\mathbf{P}_{\mathbf{i j k}} \cdot\left[\begin{array}{ccc}
d_{i} & 0 & 0 \\
0 & d_{j} & 0 \\
0 & 0 & d_{k}
\end{array}\right] \cdot \mathbf{P}_{\mathbf{i j k}}^{\mathbf{T}}=\mathbf{P}_{\mathbf{i j k}} \mathbf{Q}_{\mathbf{3}} \mathbf{P}_{\mathbf{i j k}}^{\mathbf{T}}
$$

where $\mathbf{P}_{\mathbf{i j k}}$ is a permutation matrix possibly necessary to order the eigenvalues in the diagonal. Substituting equation 12 into the equation of $\mathrm{Q}_{\mathbf{3}_{\text {cam }}}$ it then holds that:

$$
\mathbf{R}=\left(\mathbf{V}_{\mathbf{3}} \mathbf{P}_{\mathbf{i j k}}\right)^{\mathbf{T}} \quad \mathbf{Q}_{\mathbf{3}}=\left[\begin{array}{lll}
1 & 0 & 0 \\
0 & 1 & 0 \\
0 & 0 & d
\end{array}\right]
$$

which gives the estimates for the rotation matrix and the first $3 \times 3$ block of the quadric, where since the quadric is rotationally symmetric, the first two diagonal elements are equal and can be made, by scaling, equal to 1 . For the remaining unknowns (translation vector $\mathbf{t}$ and $q_{44}$ ), the elements $\mathbf{q}_{\text {cam }}$ e $q_{44}$ cam are used.

However, different analysis are made for hyperbolic, elliptic and spherical mirrors and for parabolic mirrors. Consider now the first mirror type in which case $\mathbf{q}=\mathbf{0}^{T}$. Therefore:

$$
\mathbf{t}=\mathbf{Q}_{3}^{-1} \mathbf{R}^{-\mathrm{T}} \mathbf{q}_{\text {cam }}
$$

and

$$
q_{44}=q_{44} \text { cam }-\mathbf{t}^{\mathbf{T}} \mathbf{Q}_{\mathbf{3}} \mathbf{t}
$$

which completes the estimation of the pose of the camera relative to the quadric mirror for hyperbolic, elliptic and spherical mirrors.

Consider now the case of parabolic mirrors in which case $B \neq 0$ and $A=0 ; C=0$. In this case the diagonal matrix $\mathbf{Q}_{3}$ has only two nonzero elements and so its identification is easy. Expanding now the elements of $\mathbf{q}_{\mathbf{c a m}}$ the following three linear equations are obtained:

$$
\left\{\begin{array}{l}
r_{11} t_{1}+r_{21} t_{2}+r_{31} \frac{B}{2}=q_{1 c a m} \\
r_{12} t_{1}+r_{22} t_{2}+r_{32} \frac{B}{2}=q_{2_{c a m}} \\
r_{13} t_{1}+r_{23} t_{2}+r_{33} \frac{B}{2}=q_{3 c a m}
\end{array} \Longleftrightarrow\left[\begin{array}{c}
t_{1} \\
t_{2} \\
\frac{B}{2}
\end{array}\right]=\mathbf{R}^{-\mathbf{T}} \mathbf{q}_{\mathbf{c a m}}\right.
$$

and finally, expanding the equation for $q_{44 \text { cam }}$, the equation to estimate the last unknown is: 


$$
t_{3}=\frac{q_{44_{c a m}}-t_{1}^{2}-t_{2}^{2}}{B}
$$

since $q_{44}=0$.

We have therefore shown that it is possible to recover linearly the pose of the mirror relative to the camera and the misalignment of both (as long as the quadric matrix is known in the camera coordinate system).

\section{EXPERIMENTAL RESULTS}

In this section we show the usefulness of the method described by applying it to synthetic data and also to real images. We estimate the quadric that describes the mirror surface, its pose relative to the camera and the pose of the camera relative to the world reference frame.

We start by presenting the results with synthetic data. Two different camera/mirror configurations are considered: a perspective camera with a spherical mirror with a $37.5 \mathrm{~mm}$ radius (the camera and the mirror are not aligned) and an orthographic camera with a parabolic mirror in a paracatadioptric configuration (the axis is aligned with the mirror).

The algorithm tries to minimize the error function using two known minimization methods that are combined: a genetic algorithm and the simplex Nelder-Mead method. Successive runs of both methods, one at a time, led to solutions.

Convergence was tested in two different cases. In the first case we added some noise (gaussian distribution with zero mean) to the ground truth values. The algorithm was then run until one of the stopping conditions was achieved: (1) the error was smaller than a tolerance value (1e-5); (2) a predefined maximum elapsed time was reached and (3) unchanged error value for a predefined time interval (local minimum).

In the second case we start the experiment by using a random vector as the initial values for the parameters to be estimated. We performed several runs of the algorithm, usually between 10 and 100, using different random starting values and in general when the stopping conditions are met the estimates obtained are close to the optimal values. Next, these results are used to rerun the genetic algorithm as starting chromosomes. The algorithm was iterated until one of the stopping conditions was met.

We noticed no difference between the results in both tests. This indicates that the convergence is in general obtained irrespective of using or not values close to the ground truth. 
Table I presents the values obtained for the parameters and the corresponding ground truth values as well as the angle and amplitude errors for the estimated and true vectors (perspective camera with spherical mirror). Table II presents the same analysis for the paracatadioptric camera.

As it can be seen from the results with synthetic data, the quadric in the camera coordinates $\mathrm{Q}_{\text {cam }}$ (which integrates both the quadric $\mathrm{Q}$ in the canonical form and the pose $\mathbf{T}$ ) and the pose $\mathbf{H}$ are estimated accurately. The estimates for the actual values of $\mathbf{Q}$ and $\mathbf{T}$ are not computed for synthetic data since the equations presented are in closed form and their values are highly accurate. They are however computed in the experiments with real images.

In the experiments with real images, we present the results obtained with a non-central catadioptric camera made up by a perspective camera and a spherical mirror. The same camera is also used in a hyperbolic configuration. The camera used was a commercial CANON EOS 350D with image size 3456x2304. The spherical mirror has a radius of $37.5 \mathrm{~mm}$. The systems were previously calibrated using a two step algorithm by first estimating the perspective camera parameters and then calibrating the mirror. The mirrors were not perfectly aligned with the camera. Figure 2 displays an image acquired by both cameras.

The calibration object used to calibrate the camera and to test the algorithm can be planar or non-planar. Non-planar calibration objects usually yield better calibration results since their nonplanar structure inherently provides additional information. However, tests performed with planar patches proved to provide estimates with good accuracy. Planar patterns have the advantage of simplicity. We chose to use non-planar objects in the experiments with the spherical mirror tests and planar patterns in the experiments with the hyperbolic mirror.

First we present the results concerning the spherical mirror configuration. Since in the case of hyperbolic mirror the apparent contour of the quadric mirror is not visible (because the mirror is a hyperboloid cut by a plane) we present the results without using the apparent contour information. As in the case of synthetic data, the accuracy of the method is tested in two ways namely by using initial values for the parameters that are obtained by adding some noise to the true values and also by starting the iterations with random values for the parameters. Monte Carlo methods are applied and the results obtained are presented in table III for the case of the spherical mirror using the apparent contour and in table IV without using the apparent contour.

Using the hyperbolic mirror, the experiments are similar and their results are presented in table $\mathrm{V}$.

The results obtained in the experiments with real images show that estimates for the quadric 
mirror parameters in the camera coordinate system $\left(\mathrm{Q}_{\text {cam }}\right)$ and for the the pose of the camera in the world reference frame $(\mathbf{H})$ can be obtained with good accuracy. The use of the apparent contour allows for a drastic improvement of the accuracy of the estimates. The next step is the estimation of the parameters of the quadric mirror in its canonical form $(\mathrm{Q})$ and the estimation of its pose relative to the camera - $(\mathbf{T})$. For that purpose we used the equations of section V. Since the mirror is a sphere, the meaningful parameters are the translation between the camera and the center of the mirror and its radius. Tables VI and VII show the results obtained for the parameters using and not using the apparent contour respectively. The rotation matrix $\mathbf{R}$ is not shown since due to the nature of the mirror any other rotation would express the same mirror (it is meaningless the orientation of a spherical mirror).

From the results one can conclude that the radius of the mirror surface is obtained with high accuracy in all cases where the apparent contour is used. In the cases where the apparent contour is not used accurate estimates for the radius can only be obtained if the initial values for the parameters are close to their optimal values.

For the hyperbolic mirror, the results for the estimates of the quadric mirror parameters and for its pose relative to the camera are presented in table VIII.

It should be remarked that the experimental results show that a good estimate for the conic corresponding to the apparent contour of the mirror is very important for the accuracy of the results. This is the most sensitive set of parameters and small errors in their values cause high errors in the estimation of all the other parameters.

\section{Reprojection error}

In order to have an exact idea of how erroneous is the estimation and what implications it does have in the calibrated model, we reproject the 3D points to the image and compare the actual and recomputed pixel locations. Figure 3 shows some cases of reprojected points and the actual pixels, using some results obtained with the spherical mirror, with and without the apparent contour information.

Table IX presents some additional cases and the sum and average errors per pixel. Table IX presents results obtained with all the spherical mirror data (contour and no contour) and with the hyperbolic mirror. The corresponding amplitude and angle error of the estimated state vector is presented. 
As seen in table IX, the reprojection error per pixel grows with the error in the estimated values (the poses of the camera relative to the mirror and to world reference frame and also the mirror parameters).

\section{SUMMARY AND CONCLUSIONS}

In this paper we presented a method to calibrate catadioptric camera systems made up by a perspective or orthographic camera (whose internal parameters are considered to be known) and a curved mirror whose shape is mathematically expressed by a non-degenerate quadric (includes spheres, hyperbolic, parabolic and elliptic mirrors). The method requires the knowledge of the intrinsic parameters of the camera and also local world calibration information (for instance distances in a calibration pattern).

The method allows the use of the apparent contour of the mirror (which is a conic) to constrain the quadric mirror and its pose relative to the camera and then applies a nonlinear iterative minimization method to match some back projected pattern points to a $3 \mathrm{D}$ grid. This method first estimates the pose of the camera relative to the world reference frame and the quadric mirror in camera coordinates. In the second step, using closed form expressions, it estimates the camera in its canonical form and its pose.

Experimental results showed that the method is accurate both with synthetic data and with real images, even when the initial estimates (required by the non-linear optimization procedure) are completely random, specially if the apparent contour is used. It was also concluded that the estimation of the conic parameters corresponding to the mirror apparent contour is critical to the accuracy of the results.

Future directions of this work include quantization of the error propagation from the estimates of parameters of the conic of the apparent contour to the results.

\section{Acknowledgments}

The authors gratefully acknowledge the support of projects OMNISYSPOSI / SRI/41506/2001 and CAM IDESPOSI/SRI/45970/2002, funded by the Portuguese 
Foundation for Science and Technology and FEDER.

[1] S. Nayar and S. Baker, "Catadioptric Image Formation", DARPA Image Understanding Workshop, New Orleans (May 1997)

[2] S. Baker and S. Nayar, "A Theory of Catadioptric Image Formation", IEEE International Conference on Computer Vision, 35-42, Bombay (1998)

[3] D. Burkhard and D. Shealy, "Flux density for ray propagation in geometrical optics", Journal of the Optical Society of America, 63(3), 299-304 (1973)

[4] R. Swaminathan, M. Grossberg and S. Nayar, "Caustics of Catadioptric Cameras", IEEE International Conference on Computer Vision, Vancouver (Jul 2001)

[5] E. Hecht, Optics, Addison-Wesley (1987)

[6] C. Geyer and K. Daniilidis, "A Unifying Theory for Central Panoramic and Practical Implications", European Conference on Computer Vision, 445-461, Dblin (2000)

[7] C. Geyer and K. Daniilidis, "Catadioptric Projective Geometry", IJCV, 45(3), 223-243 (2001)

[8] J. Barreto and H. Araujo, "Issues on the Geometry of Central Catadioptric Imaging", CVPR2001 International Conference on Pattern Recognition, Haway, USA (2001)

[9] C. Geyer and K. Daniilidis, "Properties of the Catadioptric Fundamental Matrix", European Conference on Computer Vision, 140-154 (2002)

[10] T. Svoboda and T. Pajdla, "Epipolar Geometry for Central Catadioptric Cameras", IJCV, 49(1), 23-37 (Aug. 2002)

[11] J. Gluckman and S. Nayar, "Rectified Catadioptric Stereo Sensors", IEEE Conference on Computer Vision and Pattern Recognition, June (2000)

[12] Y. Pritch, M. Ben-Ezra and Shmuel Peleg, Optics for OmniStereo Imaging, 447-467, Kluwer Academic (2001)

[13] J. Yu and L. McMillan, "General Linear Cameras", 8th European Conference on Computer Vision, LNCS (3022), May (2004)

[14] M. Grossberg and S. Nayar, "A General Imaging Model and a Method for Finding its Parameters", IEEE International Conference on Computer Vision, Vancouver (Jul 2001)

[15] R. Pless, "Using Many Cameras as One", IEEE Conference on Computer Vision and Pattern Recognition (2003) 
[16] P. Sturm and S. Ramalingam, "A Generic Concept for Camera Calibration", Europeen Conference on Computer Vision, Prague (May 2004)

[17] S. Ramalingam, S. Lodha and P. Sturm", "A Generic Structure-from-Motion Algorithm for CrossCamera Scenarios", 5th Workshop on Omnidirectional Vision, Camera Networks and Non-classical Cameras", 175-186 (May 2004)

[18] S. Ramalingam, P. Sturm and S. Lodha, "Towards Complete Generic Camera Calibration", IEEE International Conference on Computer Vision, Beijing (Oct 2005)

[19] P. Sturm, Multi-View Geometry for General Camera Models, IEEE International Conference on Computer Vision, Beijing (Oct 2005)

[20] C. Chen and W. Chang, "On Pose Recovery for Generalized Visual Sensors", IEEE Transactions on Pattern Analysis and Machine Intelligence, 26(7), 848-861 (July 2004)

[21] K. Torrence and E. Sparrow, ”Theory for Off-Specular Reflection From Roughened Surfaces”, Journal of the Optical Society of America, 57(9), 1105-1114 (Sep 1967)

[22] G. Healey and T. Binford, "Local Shape from Specularity", Computer Vision, Graphics and Image Processing, 42(1), 62-86 (April 1988)

[23] J. Koenderink and A. Doorn, "Photometric Invariants Related to Solid Shape”, Optica Acta, 27(7), 981-996 (1980)

[24] K. Ikeuchi, 'Determinig Surface Orientations of Specular Surfaces by Using the Photometric Stereo Method", IEEE Transactions on Pattern Analysis and Machine Intelligence, 3(6), 661-669 (November 1981)

[25] S. Nayar, K. Ikeuchi and T. Kanade, ”Determinig Shape And Reflectance of Hybrid Surface by Photometric Sampling”, IEEE Transactions on Robotics and Automation, 6(4), 418-431 (August 1990)

[26] S. Rahmann and N. Canterakis, "Reconstruction of Specular Surfaces using Polarization Imaging", Conference on Computer Vision and Pattern Recognition (2001)

[27] S. Nayar, X. Fang and T. Boult, "Separation of Reflection Components Using Color and Polarization”, International Journal of Computer Vision, 21(3), 163-186 (February 1997)

[28] A. Sanderson, L. Weiss and S. Nayar, "Structured Highlight Inspection of Specular Surfaces", IEEE Transactions on Pattern Analysis and Machine Intelligence, 10(1), 44-55 (January 1988)

[29] J. Neumann, C. Fermüller, and Y. Aloimonos, "Eye Design in the Plenoptic Space of Light Rays", Ninth IEEE International Conference on Computer Vision, Nice (Oct 2001)

[30] S. Savarese, M. Chen and P. Perona, "Recovering local shape of a mirror surface from reflection of a 
regular gridI", European Conference of Computer Vision, Prague (May 2004)

[31] H. Schultz, "Retrieving Shape Information from Multiple Images of a Specular Surface", IEEE Transactions on Pattern Analysis and Machine Intelligence, 16(2), 195-200 (Feb. 1994)

[32] M. Longuet-Higgins, "Reflection and Refraction at a Random Moving Surface: i, ii and iii", Journal of the Optical Society of America, 50(9), 838-856 (September 1960)

[33] M. Oren and S. Nayar, "A Theory of Specular Surface Geometry", International Journal of Computer Vision, 24(2), 105-124 (1996)

[34] X. Ying and Z. Hu, "Catadioptric Camera Calibration Using Geometric Invariants", IEEE Transactions on Pattern Analysis and Machine Intelligence, 26(10), 1260-1271 (Oct 2004)

[35] Y. Wu and Z. Hu, "Geometric Invariants and Applications under Catadioptric Camera Model", International Conference on Computer Vision, Beijing (Oct 2005)

[36] D.G. Aliaga, ”Accurate Catadioptric Calibration For Real-Time Pose Estimation In Room-Size Environments", 8th International Conference on Computer Vision, 127-134 (2001)

[37] J. Fabrizio, J.-P. Tarel and R. Benosman, "Calibration of Panoramic Catadioptric Sensors Made Easier", Workshop on Omnidirectional Vision, Camera Networks and Non-classical Cameras, 45-52 (2002)

[38] B. Micusík and T. Pajdla, ”Autocalibration and 3D Reconstruction with Non-central Catadioptric Cameras", Conference on Computer Vision and Pattern Recognition, Washington US, (Jun 2004)

[39] T. Pajdla, "Stereo with Oblique Cameras", IJCV, 47(3), 161-170 (April 2002)

[40] S.-W. Jeng and W.-H. Tsai", "Precise Image Unwarping of Omnidirectional Cameras with HyperbolicShaped Mirrors", 16th Conference on Computer Vision, Graphics and Image Processing, 414-422 (2003)

[41] S.-W. Jeng and W.-H. Tsai, "Construction of Perspective and Panoramic Images from Omni-images Taken from Hypercatadioptric Cameras for Visual Surveillance", International Conference on Networking, Sensing and Control, Taiwan, 204-209, (2004)

[42] D. Strelow and J.S. Mishler and S. Singh and H. Herman, "Extending Shape from Motion to NonCentral Omnidirectional Cameras", International Conference on Intelligent Robots and Systems (Oct 2001)

[43] N. Gonçalves and H. Araujo, "Estimating Parameters of Non Central Catadioptric Systems Using Bundle Adjustment”, OMNIVIS'2005 - The 6th Workshop on Omnidirectional Vision, Camera Networks and Non-classical Cameras, Beijing (Oct 2005) 
[44] N. Gonçalves and H. Araujo", "Partial Calibration and Mirror Shape Recovery for Non-Central Catadioptric Systems”, OMNIVIS'2005 - 6th Workshop on Omnidirectional Vision, Camera Networks and Non-classical Cameras, Beijing (Oct 2005)

[45] R. Hartley and A. Zisserman, Multiple View Geometry in Computer Vision, Cambridge University Press (2000)

[46] L. Dupont, D. Lazard, S. Lazard and S. Petitjean, "Near-Optimal Parameterization of the Intersection of Quadrics", Annual Symposium on Computational Geometry, San Diego, USA, 246-255 (June 2003)

[47] L. Dupont, D. Lazard, S. Lazard and S. Petitjean, "Intersecting Quadrics: An Efficient and Exact Implemetation", Annual Symposium on Computational Geometry New York, Usa, 419-425 (2004)

[48] F. Uhlig, "Simultaneous Block Diagonalization of Two Real Symmetric Matrices", Linear Algebra and Its Applications, 7(4), 281-289 (1973)

[49] F. Uhlig, "A Canonical Form For a Pair of Real Symmetric Matrices That Generate a Nonsingular Pencil”, Linear Algebra and Its Applications, 14, 189-209 (1976)

[50] M. Born and E. Wolf, Principles of Optics, Pergamon Press (1965)

[51] J. Semple and G. Kneebone, Algebraic Projective Geometry, Oxford University Press, London (1959)

[52] J. Stolfi, Oriented Projective Geometry, Academic Press (1991)

Helder Araujo is currently Full Professor at the Department of Electrical and Computer Engineering, University of Coimbra, Portugal. He is co-founder of the Portuguese Institute for Systems and Robotics (ISR), where he is currently a Researcher and Vice-Director of the Coimbra site. His primary research interests are in computer vision and cognitive robotics. [Figure 4]

Nuno Gonçalves is concluding his $\mathrm{PhD}$ degree at the University of Coimbra doing his research at the Institute for Systems and Robotics (ISR-Coimbra). His current work is on Computer Vision, on the study of geometrical properties and calibration of non central catadioptric cameras. He is Teaching Assistant in the Department of Electrical and Computer Engineering, University of Coimbra, Portugal. His main scientific interests include computer vision, optics, robotics and medical imaging. [Figure 5] 
TABLE I: Tests with synthetic data for a perspective camera with an off-axis spherical mirror configuration. Notice that the parameters of the error random distribution are relative to the ground truth values of the parameters, that is, for instance error between 0 and 1 is error between 0 and $100 \%$ of the true value.

\begin{tabular}{|c|c|c|c|c|c|c|c|c|c|c|c|c|}
\hline & $q_{14}$ & $q_{24}$ & $q_{33}$ & $q_{34}$ & $\theta_{X}$ & $\theta_{Y}$ & $\theta_{Z}$ & $t_{X}$ & $t_{Y}$ & $t_{Z}$ & Amp.err & Ang.err \\
\hline GT & 32.0 & 16.0 & -150.0 & $2.000 \mathrm{e} 4$ & 0.0 & 0.0 & 0.0 & 0.0 & 0.0 & -300.0 & & \\
\hline $\begin{array}{l}\mathrm{GT}+\operatorname{err}(\sim \\
N(0 ; 0.5))\end{array}$ & 33.18 & 16.83 & -147.14 & $2.190 \mathrm{e} 4$ & -0.01 & -0.00 & 0.00 & 0.00 & 0.01 & -296.84 & 0.86 & 0.00 \\
\hline $\begin{array}{l}\text { Random } \\
(\sim \operatorname{Unif}(0 ; 1))\end{array}$ & 31.78 & 15.28 & -149.95 & $2.247 \mathrm{e} 4$ & -0.01 & 0.01 & 0.01 & -0.00 & 0.00 & -303.14 & 1.70 & 0.01 \\
\hline $\begin{array}{l}\text { Random } \\
(\sim \operatorname{Unif}(0 ; 2))\end{array}$ & 32.23 & 17.31 & -152.48 & $2.256 \mathrm{e} 4$ & 0.01 & 0.01 & 0.01 & -0.01 & -0.00 & -298.59 & 2.07 & 0.02 \\
\hline $\begin{array}{l}\text { Random } \\
(\sim \operatorname{Unif}(-1 ; 1))\end{array}$ & 33.23 & 17.36 & -153.04 & $2.146 \mathrm{e} 4$ & 0.00 & 0.00 & -0.01 & -0.01 & 0.01 & -298.82 & 2.88 & 0.03 \\
\hline $\begin{array}{l}\text { Random } \\
(\sim U n i f(-2 ; 2))\end{array}$ & 31.06 & 17.09 & -144.10 & $2.301 \mathrm{e} 4$ & -0.01 & 0.01 & 0.00 & -0.01 & -0.02 & -299.47 & 4.11 & 0.04 \\
\hline
\end{tabular}


TABLE II: Tests with synthetic data. The configuration is made up of an orthographic camera with an aligned parabolic mirror. Notice that the parameters of the error random distribution are relative to the ground truth values of the parameters, that is, for instance error between 0 and 1 is error between 0 and $100 \%$ of the true value.

\begin{tabular}{|c|c|c|c|c|c|c|c|c|c|c|c|c|}
\hline & $q_{14}$ & $q_{24}$ & $q_{33}$ & $q_{34}$ & $\theta_{X}$ & $\theta_{Y}$ & $\theta_{Z}$ & $t_{X}$ & $t_{Y}$ & $t_{Z}$ & Amp.err & Ang.err \\
\hline GT & 0.0 & 0.0 & -600.0 & $1.800 \mathrm{e} 5$ & 0.0 & 0.0 & 0.0 & 0.0 & 0.0 & -300.0 & & \\
\hline $\begin{array}{l}\mathrm{GT}+\operatorname{err}(\sim \\
N(0 ; 0.5))\end{array}$ & 0.00 & 0.01 & -587.39 & $1.785 \mathrm{e} 5$ & -0.00 & -0.01 & -0.01 & 0.01 & 0.00 & -296.52 & 0.83 & 0.00 \\
\hline $\begin{array}{l}\text { Random } \\
(\sim \operatorname{Unif}(0 ; 1))\end{array}$ & -0.00 & -0.00 & -605.24 & $1.781 \mathrm{e} 5$ & -0.00 & -0.00 & -0.00 & 0.02 & -0.01 & -292.72 & 1.07 & 0.00 \\
\hline $\begin{array}{l}\text { Random } \\
(\sim U n i f(0 ; 2))\end{array}$ & 0.00 & -0.01 & -600.39 & $1.780 \mathrm{e} 5$ & 0.01 & 0.01 & -0.00 & -0.02 & 0.00 & -308.60 & 1.13 & 0.00 \\
\hline $\begin{array}{l}\text { Random } \\
(\sim U n i f(-1 ; 1))\end{array}$ & 0.01 & -0.02 & -599.15 & $1.778 \mathrm{e} 5$ & -0.01 & -0.01 & 0.00 & -0.00 & 0.00 & -298.01 & 1.23 & 0.00 \\
\hline $\begin{array}{l}\text { Random } \\
(\sim U n i f(-2 ; 2))\end{array}$ & 0.01 & 0.00 & -589.31 & $1.843 \mathrm{e} 5$ & 0.00 & 0.01 & 0.01 & -0.01 & -0.01 & -299.61 & 2.41 & 0.01 \\
\hline
\end{tabular}


TABLE III: Tests with real images obtained from a perspective camera with a spherical mirror. Estimated values for the quadric mirror in the camera coordinate system - $Q_{c a m}$ and for the pose of the camera relative to the world reference frame - $H$, using the apparent contour to reduce the uncertainty.

\begin{tabular}{|c|c|c|c|c|c|c|c|c|c|c|c|c|}
\hline & $q_{14}$ & $q_{24}$ & $q_{33}$ & $q_{34}$ & $\theta_{X}$ & $\theta_{Y}$ & $\theta_{Z}$ & $t_{X}$ & $t_{Y}$ & $t_{Z}$ & Amp.err & Ang.err \\
\hline GT & -0.18 & 11.67 & -272.46 & 7.296e4 & 0.00 & 0.00 & 0.00 & 50.00 & 50.00 & -300.00 & & \\
\hline $\begin{array}{l}\mathrm{GT}+\operatorname{err}(\sim \\
N(0 ; 0.5))\end{array}$ & 0.01 & 11.75 & -269.54 & 7.140e4 & -0.00 & -0.00 & 0.00 & 50.71 & 50.41 & -302.60 & 2.15 & 0.01 \\
\hline $\begin{array}{l}\text { Random } \\
(\sim U n i f(0 ; 1))\end{array}$ & 0.56 & 9.97 & -269.30 & $7.128 \mathrm{e} 4$ & 0.00 & -0.00 & 0.00 & 51.90 & 49.47 & -303.18 & 2.31 & 0.01 \\
\hline $\begin{array}{l}\text { Random } \\
(\sim U n i f(0 ; 2))\end{array}$ & 0.64 & 10.41 & -280.18 & 7.716e4 & 0.00 & -0.00 & 0.00 & 51.97 & 49.08 & -293.31 & 5.74 & 0.02 \\
\hline $\begin{array}{l}\text { Random } \\
(\sim \operatorname{Unif}(-1 ; 1))\end{array}$ & 0.17 & 11.30 & -286.35 & $8.056 \mathrm{e} 4$ & -0.00 & -0.00 & 0.00 & 50.80 & 49.77 & -287.40 & 10.44 & 0.03 \\
\hline $\begin{array}{l}\text { Random } \\
(\sim U n i f(-2 ; 2))\end{array}$ & 0.16 & 11.04 & -279.64 & 7.686e4 & -0.00 & -0.00 & 0.00 & 50.83 & 50.04 & -293.53 & 5.33 & 0.02 \\
\hline
\end{tabular}


TABLE IV: Tests with real images obtained from a perspective camera with a spherical mirror. Estimated values for the quadric mirror in the camera coordinate system - $Q_{c a m}$ and for the pose of the camera relative to the world reference frame - $H$, not using the apparent contour. Notice that when the apparent contour is not used the algorithm only converges to the solution with good initial parameter estimates.

\begin{tabular}{|l|l|l|l|l|l|l|}
\hline & GT & GT+err & Random $~$ & Random & Random $~$ & Random $~$ \\
\hline$q_{11}$ & 1.00 & 1.00 & -4.18 & -51.86 & 0.97 & -11.59 \\
\hline$q_{12}$ & 0.00 & -0.00 & 0.36 & -0.41 & -0.00 & 0.37 \\
\hline$q_{13}$ & 0.00 & 0.00 & 1.21 & -1.34 & 0.00 & 1.75 \\
\hline$q_{14}$ & -0.18 & -0.19 & 0.72 & -37.40 & -0.28 & 229.81 \\
\hline$q_{22}$ & 1.00 & 1.00 & -4.63 & -53.23 & 0.97 & -12.10 \\
\hline$q_{23}$ & 0.00 & 0.00 & 1.13 & -1.92 & 0.00 & 2.16 \\
\hline$q_{24}$ & 11.67 & 11.50 & 0.92 & 44.17 & 7.74 & 404.01 \\
\hline$q_{34}$ & -272.46 & -268.90 & 0.11 & -268.98 & -187.28 & -161.46 \\
\hline$q_{44}$ & 72964.16 & 71074.54 & -0.30 & -22139.85 & 34502.16 & -66674.35 \\
\hline$\theta_{1}$ & 0.00 & 0.00 & -0.08 & 3.02 & 0.00 & -0.08 \\
\hline$\theta_{2}$ & 0.00 & 0.00 & 0.21 & -3.03 & 0.00 & 0.08 \\
\hline$\theta_{3}$ & 0.00 & 0.00 & 0.03 & -3.13 & 0.00 & 0.01 \\
\hline$t_{1}$ & 50.00 & 49.99 & -34.41 & 108.32 & 49.74 & 20.44 \\
\hline$t_{2}$ & 50.00 & 50.12 & 107.27 & 122.11 & 52.95 & 111.54 \\
\hline$t_{3}$ & -300.00 & -303.25 & -505.44 & -551.32 & -378.04 & -663.26 \\
\hline Amp. err & - & 2.59 & 99.29 & 69.64 & 52.71 & 8.61 \\
\hline Angle err & - & 0.01 & 89.80 & 178.03 & 0.41 & 179.02 \\
\hline
\end{tabular}


TABLE V: Tests with real images obtained from a perspective camera with a hyperbolic mirror. Estimated values for the quadric mirror in the camera coordinate system - $Q_{c a m}$ and for the pose of the camera relative to the world reference frame - $H$, without using the apparent contour.

\begin{tabular}{|l|l|l|l|l|l|l|l|}
\hline & GT & GT+err & GT+err & GT+err & GT+err & GT+err & GT+err $~$ \\
\hline$q_{11}$ & 1.00 & 1.00 & 1.01 & 0.93 & 1.59 & 2.60 & -10.06 \\
\hline$q_{12}$ & 0.00 & 0.00 & 0.00 & 0.01 & -0.03 & 0.15 & -8.24 \\
\hline$q_{13}$ & 0.00 & -0.00 & -0.00 & 0.03 & 0.07 & -0.01 & 1.16 \\
\hline$q_{14}$ & 4.11 & 4.45 & 4.48 & -1.74 & -11.67 & 11.30 & -2323.02 \\
\hline$q_{22}$ & 0.00 & -0.00 & 0.00 & -0.01 & -0.06 & -0.01 & 0.29 \\
\hline$q_{23}$ & -5.14 & -5.14 & -5.18 & -3.43 & 17.92 & -7.77 & -1480.27 \\
\hline$q_{24}$ & -0.76 & -0.76 & -0.75 & -0.73 & -0.42 & -1.12 & -0.06 \\
\hline$q_{34}$ & 138.02 & 137.82 & 137.32 & 143.04 & 80.84 & 194.75 & 166.29 \\
\hline$q_{44}$ & -24436.70 & -24358.40 & -24356.89 & -27326.14 & -10872.63 & -29178.30 & -357550.10 \\
\hline$\theta_{1}$ & 0.00 & 0.00 & -0.01 & -0.01 & -0.35 & 0.11 & -0.29 \\
\hline$\theta_{2}$ & 0.00 & 0.00 & 0.01 & -0.02 & 0.07 & 0.08 & -0.05 \\
\hline$\theta_{3}$ & 0.00 & -0.00 & 0.00 & -0.02 & 0.09 & 0.02 & 0.26 \\
\hline$t_{1}$ & 50.00 & 47.95 & 43.60 & 56.52 & 47.74 & 4.09 & -166.45 \\
\hline$t_{2}$ & 50.00 & 47.92 & 54.09 & 53.88 & 244.74 & 33.07 & 254.14 \\
\hline$t_{3}$ & -300.00 & -300.96 & -299.00 & -277.94 & -152.44 & -307.41 & -177.92 \\
\hline Amp.err & - & 0.27 & 0.29 & 2.17 & 119.12 & 55.91 & 17445.90 \\
\hline Angle err & - & 1.76 & 3.35 & 24.38 & 71.61 & 30.10 & 98.30 \\
\hline
\end{tabular}


TABLE VI: Experimental results with real images obtained by a perspective camera with a spherical mirror. Estimated values for the pose of the mirror relative to the camera - $t$ and for its radius, using the apparent contour.

\begin{tabular}{|c|c|c|c|c|c|c|c|}
\hline & $t_{1}$ & $t_{2}$ & $t_{3}$ & Amp.err & Ang.err & Radius & Amp.err \\
\hline GT & -0.1837 & 11.667 & -272.46 & - & - & 37.5 & $\bullet$ \\
\hline $\begin{array}{l}\text { GT }+\operatorname{err}(\sim \\
N(0 ; 0.5))\end{array}$ & 0.01 & 11.64 & -266.87 & 2.05 & 0.06 & 37.14 & 0.97 \\
\hline $\begin{array}{l}\text { Random } \\
(\sim U n i f(0 ; 1))\end{array}$ & 0.56 & 9.92 & -267.73 & 1.76 & 0.37 & 37.01 & 1.30 \\
\hline $\begin{array}{l}\text { Random } \\
(\sim U n i f(0 ; 2))\end{array}$ & 0.64 & 10.34 & -278.45 & 2.18 & 0.37 & 38.51 & 2.70 \\
\hline $\begin{array}{l}\text { Random } \\
(\sim \operatorname{Unif}(-1 ; 1))\end{array}$ & 0.16 & 11.22 & -284.22 & 4.30 & 0.20 & 39.45 & 5.20 \\
\hline $\begin{array}{l}\text { Random } \\
(\sim U n i f(-2 ; 2))\end{array}$ & 0.16 & 10.96 & -277.57 & 1.86 & 0.20 & 38.53 & 2.74 \\
\hline
\end{tabular}


TABLE VII: Experimental results with real images obtained by a perspective camera with a spherical mirror. Estimated values for the pose of the mirror relative to the camera - $t$ and for the radius, without using the apparent contour. Notice that when the apparent contour is not used the algorithm only converges to the solution if started with good initial estimates.

\begin{tabular}{|c|c|c|c|c|c|c|c|}
\hline & $t_{1}$ & $t_{2}$ & $t_{3}$ & Amp.err & Ang.err & Radius & Amp.err \\
\hline GT & -0.1837 & 11.667 & -272.46 & - & - & 37.5 & • \\
\hline $\begin{array}{l}\mathrm{GT}+\operatorname{err}(\sim \\
N(0 ; 0.5))\end{array}$ & -0.19 & 11.50 & -268.90 & 1.31 & 0.00 & 36.98 & 1.40 \\
\hline $\begin{array}{l}\text { Random } \\
(\sim \operatorname{Unif}(0 ; 1))\end{array}$ & 5.09 & 9.42 & -7.79 & 95.15 & 51.83 & 18.54 & 50.55 \\
\hline $\begin{array}{l}\text { Random } \\
(\sim U n i f(0 ; 2))\end{array}$ & -1.07 & 79.39 & -50.32 & 65.53 & 55.18 & 58.03 & 54.76 \\
\hline $\begin{array}{l}\text { Random } \\
(\sim \operatorname{Unif}(-1 ; 1))\end{array}$ & -0.28 & 7.74 & -187.27 & 31.27 & 0.10 & 25.19 & 32.83 \\
\hline $\begin{array}{l}\text { Random } \\
(\sim U n i f(-2 ; 2))\end{array}$ & -19.32 & 13.76 & 262.19 & 3.47 & 173.09 & 39.78 & 6.09 \\
\hline
\end{tabular}


TABLE VIII: Experimental results with real images obtained by a perspective camera with a hyperbolic mirror. Estimated values for the pose of the mirror relative to the camera $-t$ and for the radius, without using the apparent contour. Notice that when the gaussian error introduced is very high, the algorithm does not converge to an useful solution.

\begin{tabular}{|l|l|l|l||l|l|l||l|l|}
\hline & $\theta_{1}$ & $\theta_{2}$ & $\theta_{3}$ & $t_{1}$ & $t_{2}$ & $t_{3}$ & Amp.err & Ang.err \\
\hline GT & 0.0 & 0.0 & 0.0 & 4.11 & -5.12 & -181.42 & $\bullet$ & $\bullet$ \\
\hline GT+err $\sim$ & 0.00 & -0.00 & -0.40 & 4.87 & -4.49 & -182.12 & 0.39 & 0.34 \\
$N(0 ; 0.005))$ & & & & & & & & \\
\hline GT+err $(\sim$ & 0.00 & -0.00 & 0.48 & 0.83 & -7.30 & -183.33 & 1.07 & 1.24 \\
$N(0 ; 0.01))$ & -0.00 & 0.18 & -0.31 & 13.83 & 0.75 & -190.05 & 4.96 & 3.41 \\
\hline $\begin{array}{l}\text { GT+err } \sim \\
N(0 ; 0.05))\end{array}$ & 0.01 & -0.01 & 0.12 & 2.73 & -9.33 & -203.45 & 12.19 & 1.14 \\
\hline $\begin{array}{l}\text { GT+err }(\sim \\
N(0 ; 0.1))\end{array}$ & 0.77 & -1.40 & 0.88 & 2.02 & 54.50 & 0.44 & 69.94 & 92.04 \\
\hline GT+err $(\sim$ \\
$N(0 ; 0.5))$
\end{tabular}


TABLE IX: Reprojection error of 3D points (in pixels) and the corresponding amplitude error (in percentage) and angle errors (in degrees) in the estimated state vectors.

\begin{tabular}{|l|l|l|l|l|l|}
\hline Mirror & Contour & Initial guess & Amplitude error & Angle error & Reproj. error \\
\hline Spherical & Yes & GT+N $(0 ; 0.5)$ & 0.15 & 0.16 & 0.01 \\
\hline Spherical & No & GT+N(0;0.5) & 1.34 & 1.46 & 0.03 \\
\hline Spherical & Yes & Random $\in[0,1]$ & 0.28 & 0.32 & 0.02 \\
\hline Spherical & No & Random $\in[0,1]$ & 525.43 & 92.62 & 61.18 \\
\hline Spherical & Yes & Random $\in[0,2]$ & 1.83 & 2.16 & 0.16 \\
\hline Spherical & No & Random $\in[0,2]$ & 626.60 & 74.87 & 62.81 \\
\hline Hyperbolic & No & GT+N(0;0.005) & 0.36 & 15.48 & 0.02 \\
\hline Hyperbolic & No & GT+N(0;0.01) & 3.99 & 6.66 & 0.04 \\
\hline Hyperbolic & No & GT+N(0;0.05) & 27.14 & 37.55 & 0.13 \\
\hline Hyperbolic & No & GT+N(0;0.1) & 119.12 & 71.61 & 0.38 \\
\hline Hyperbolic & No & GT+N(0;0.5) & 502.50 & 89.74 & 9.98 \\
\hline Hyperbolic & No & GT+N(0;1.0) & 385.62 & 66.98 & 12.00 \\
\hline
\end{tabular}




\section{List of figure captions}

- Figure 1 - Coordinate systems of the camera, world and mirror and their relative positions.

- Figure 2 - Real images used.

- Figure 3 - Reprojection in the image of the 3D points. The left column presents results obtained using the apparent contour and the right column the results obtained without using of the contour. In (a)-(b) the initial estimate is the ground truth vector with gaussian noise added, with standard deviation of $50 \%$ of the actual value, in (c)-(d) the initial estimate is a random vector between 0 and $1(0 \rightarrow 100 \%$ of the actual value) and in (e)-(f) the initial estimate is a random vector between -1 and $1(-100 \% \rightarrow 100 \%$ of the actual value). 